www.jmscr.igmpublication.org Impact Factor 5.244

Index Copernicus Value: 83.27 ISSN (e)-2347-176x ISSN (p) 2455-0450 crossref DOI: _https://dx.doi.org/10.18535/jmscr/v4i11.42

\title{
Novel Thiazoles Derivatives Containing Methoxy-Napthyl Moiety as Potent Anti-Bacterial and Anti-Tubercular Agents and Its Characterization
}

\author{
Authors \\ V. J .Chandraprabha*1, D. Jagadeesh Prasad ${ }^{2}$, Kumar.C ${ }^{3}$, Prashantha Nayak ${ }^{4}$ \\ ${ }_{1,2}$ Department of Chemistry, Mangalore University, Mangalagangotri, Karnataka-574199, \\ ${ }^{3}$ Department of Bioscience, Mangalore University, Mangalagangotri, Karnataka-574199, India \\ *Corresponding Author \\ Chandaprabha.V.J \\ Email: cpjain88@yahoo.co.in,Mob: 9448062586
}

\begin{abstract}
Thiazoles and their derivatives have attracted continuing interest in both pharmaceutical and agrochemical industries and shows significant importance for the discovery of potent bioactive agents due to their various biological activities.

The present study reports the synthesis of novel 2-\{2-[(6-methoxynaphthalen-2-yl) methylidene] hydrazinyl $\}$ 4-(aryl)-1, 3-thiazole (5a-5j) which was synthesized by acid hydrolysis of 6-methoxynaphthalene-2carbaldehyde (1) and hydrazinecarbothioamide (2) which was refluxed for around 10 hours using alcohol as solvent, to yield (2E)-2-[(6-methoxynaphthalen-2-yl) methylidene] hydrazinecarbothioamide (Thiosemicarbozone) (3). The compound (3) was then condensed with different substituted phenacyl bromide (4) at $90^{\circ} \mathrm{C}$ for 8-9 hours. The structures of newly synthesized compounds were characterized by $I R,{ }^{1} H$-NMR, ${ }^{13} \mathrm{C}$-NMR and mass spectroscopic studies, few of the synthesized compounds showed moderate anti-TB activities and compounds (5d) showed moderate activity against M.tuberculosis (H37 RV Strain).Among the compounds screened for antibacterial activity (5c), (5e), (5g) and (5h) showed excellent antibacterial activity.

Keywords: 1, 3-thiazoles, Anti-bacterial and Anti-tubercular activity studies.
\end{abstract}

Thiazoles are the compounds with hetero atoms like sulphur and nitrogen and refer to a large family of derivatives. The compounds that contain thiazole moiety are synthesized from decades as they are found to exhibit various potential biological activities the structural modifications of these chemical scaffolds of 2-[(2E)-b]-4-(4methoxyphenyl)-1,3-thiazole lead discovery of novel compound with enhanced pharmacological activity. The heterocyclic scaffolds having carbothioamide has not reported and hence a decision to synthesize novel compounds bearing this moiety had made and recent literature reviews that compounds with thiazole moiety are continuously drawing the interest in the field of research, as they are found in many potent biologically active compounds and exhibits broad range of pharmacological activity such as antiinflammatory ${ }^{[1]}$, anticancer ${ }^{[2]}$, anti-protozoa ${ }^{[3]}$, anti-oxidant ${ }^{[4]}$, anti-tryposomal ${ }^{[5]}$, Neuroprotective agents ${ }^{[6]}$, anti-breast cancer ${ }^{[7]}$, anti- 
microbial $^{[8]}$, , anti-tumour ${ }^{[9,10]}$, analgesic ${ }^{[11]}$ and anticonvulsants ${ }^{[12]}$.

Hence inspired from these reports from the present review of literature a plan to synthesize novel compounds bearing thiazole moiety $(5 \mathrm{a}-5 \mathrm{j})$ was taken up in this study.

\section{MATERIALS AND METHODS}

The reaction is carried out according to well defined procedure from the detailed review of literature ${ }^{[13-19]}$. The ((2E)-2-[(6-methoxynaphthalen-2-yl) methylidene] hydrazinecarbo-thioamide (Thiosemicarbozone) (3) was synthesized by acid hydrolysis of 6-methoxynaphthalene-2carbaldehyde (1) and hydrazinecarbothioamide (2) which was refluxed for around 10 hours using alcohol as solvent, which was later condensed with different substituted phenacyl bromide (4) at $90^{\circ} \mathrm{C}$ for $8-9$ hours to yield series of novel 2-\{2[(6-methoxynaphthalen-2-yl) methylidene] hydrazine-yl $\}-4$-(aryl)-1,3-thiazole $(5 \mathrm{a}-5 \mathrm{j}) \quad$.The structures of these novel compounds $(5 \mathrm{a}-5 \mathrm{j})$ were confirmed through spectral analysis .

General procedure for the synthesis of $((2 E)-2-$ [(6-methoxynaphthalen-2-yl) methylidene] hydrazine-carbothioamide (3) :

The ((2E)-2-[(6-methoxynaphthalen-2-yl) methylidene] hydrazinecarbothioamide (Thiosemicarbozone) (3) was synthesized according to well defined procedure from the literature. The equimolar mixture of 6-methoxynaphthalene-2carbaldehyde (1) and hydrazinecarbothioamide (2) was acid hydrolyzed by refluxing the mixture for around 10 hours using alcohol as solvent .The precipitated compound was filtered and recrystallized using hot ethanol.

General procedure for the synthesis of novel 2\{2-[(6-methoxynaphthalen-2-yl) methylidene] hydrazinyl $\}-4-(\operatorname{aryl})-1,3-$ thiazole $(5 a-j)$ :

The ((2E)-2-[(6-methoxynaphthalen-2-yl) methyldene] hydrazinecarbothioamide (Thiosemicarbozone) (3) was mixed with different substituted phenacyl bromide (4) and refluxed for about 8-9 hours at $90^{\circ} \mathrm{C}$ to yield series of novel 2\{2-[(6-methoxynaphthalen-2-yl) methylidene] hydrazinyl -4-(aryl)-1,3-thiazole

(5a-j).The precipitated solid was filtered under suction, washed and recrystallized from hot ethanol.

5a

IR $\left(\mathrm{KBr}, \mathrm{Cm}^{-1}\right): 3299.59(>\underline{\mathrm{NH}}), 3112.61,2936.51(-$ $\mathrm{C}-\mathrm{H}), 1563.79(\underline{\mathrm{N}=\mathrm{C}}<$ azomethine $), 1245.90(>\underline{\mathrm{C}-\mathrm{S}-}$ ). ${ }^{13} \mathrm{C}-\mathrm{NMR}: 55.275,55.109\left(2 \underline{\mathrm{C}} \mathrm{OCH}_{3}\right), 158.781$, $151.446,140.155,134.814,129.917,129.730$ (6드 phenyl), 128.267, 127.613, 168.092 (3 thiadiazole), 145.921 (1 $\underline{\mathrm{C}}$ adjacent to naphthalene), $157.984,127.355,126.827,125.994,124.478$, $122.669,121.234,119.022,113.958,106.399$ (10 C napthyl ring). ${ }^{1} \mathrm{H}$ NMR:3.791,3.895(6H,s,2methoxy groups of naphthalene moiety and substituted benzene), $6.984 \quad(2 \mathrm{H}, \mathrm{d}, \mathrm{J}=8.4-\mathrm{p}$ methoxy benzene group), $7.356(2 \mathrm{H}, \mathrm{d}, \mathrm{J}=8.4, \mathrm{p}-$ methoxy benzene group), $7.184(1 \mathrm{H}, \mathrm{s}$, hydrogen of thiazole moiety), 7.867 (6H,m,napthoxy moiety), $8.152(1 \mathrm{H}, \mathrm{s}, \mathrm{NH}$ group adjacent to thiazole moiety), $12.016(1 \mathrm{H}, \mathrm{s}, \mathrm{CH}$ adjacent to napthoxy moiety). LC-mass: $\left[\mathrm{M}^{+}+1\right],(\mathrm{m} / \mathrm{Z}): 390.05$.

\section{$\mathbf{5 b}$}

IR $\left(\mathrm{KBr}, \mathrm{Cm}^{-1}\right)$ : 3288.66(> $\left.\underline{\mathrm{NH}}\right), 3014.78,2955.67$ $(-\mathrm{C}-\mathrm{H}), 1625.20 \quad(\underline{\mathrm{N}=\mathrm{C}}), 1270.53(>\underline{\mathrm{C}-\mathrm{S}}-) .{ }^{13} \mathrm{C}-$ NMR: 20.772, 55.268 (2 methyl and methoxy ), 157.984,136.762, 134.816, 132.046, 129.888, 129.734,(6C phenyl), 129.141,128.256, 168.111

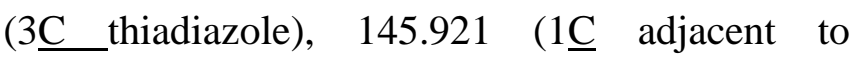
methoxy naphthalene), 151.446, 127.384 ,125.446, 124.334,123.112 , 122.653, 120.003, 119.030, 106.381, 102.605 (10 $\underline{\mathrm{C}}$ napthyl ring). ${ }^{1} \mathrm{H}$ NMR:2.326 $(3 \mathrm{H}, \mathrm{s}$, methyl group attached to benzene ring), $3.895(3 \mathrm{H}, \mathrm{s}$, methoxy groups of naphthalene moiety),7.186(4H,d,methoxy benzene), 7.352(1H,s, hydrogen of thiazole moiety) , $7.870(6 \mathrm{H}, \mathrm{m}$,napthoxy moiety) $8.163(1 \mathrm{H}, \mathrm{s}, \mathrm{NH}$ group adjacent to thiazole moiety), 12.124 $(1 \mathrm{H}, \mathrm{s}, \mathrm{CH}$ adjacent to napthoxy moiety).LCmass: $\left[\mathrm{M}^{+}+1\right],(\mathrm{m} / \mathrm{Z}): 373.02$.

5c

IR $\left(\mathrm{KBr}, \mathrm{Cm}^{-1}\right): 3309.14(>\underline{\mathrm{NH}}) 3012.74,2942.20$ C-H ), 1626.91(N=C),1269.30(>C-S-).830,728( $\underline{\text { C- }}$ 
$\underline{\mathrm{Cl}},) .{ }^{13} \mathrm{C}-\mathrm{NMR}: \quad 55.219$ (1ㅁ methoxy) ,158.437, 152.456, 140.135, 134.814, 129.917, 129.730,(6들 phenyl), 129.267, 128.613 (2C thiadiazole), 168.099, 145.921 (2C thiadiazole), 157.984, $127.355,126.827,125.994,124.478,122.669$, 121.234, 119.022, 113.958, 106.399,(10 $\underline{\mathrm{C}}$ napthyl ring). ${ }^{1} \mathrm{H}$ NMR:3.865(3H, s, methoxy groups of naphthalene moiety),6.654( $2 \mathrm{H}, \mathrm{s}$,chloro benzene), $7.763(1 \mathrm{H}, \mathrm{s}$, hydrogen of thiazole moiety), 7.729 $(6 \mathrm{H}, \mathrm{m}$,napthoxy moiety) $8.076(2 \mathrm{H}, \mathrm{d}, \mathrm{J}=8.8$ chloro benzene moiety) $8.158(1 \mathrm{H}, \mathrm{s}, \mathrm{NH}$ group adjacent to thiazole moiety),12.124(1H,s, $\mathrm{CH}$ adjacent to napthoxy moiety).LC-mass: $\left[\mathrm{M}^{+}+1\right], \quad(\mathrm{m} / \mathrm{Z})$ : 393.02/391.00.

\section{5d}

IR $\left(\mathrm{KBr}, \mathrm{Cm}^{-1}\right): 3305.05(>\underline{\mathrm{NH}}) 3015.59,2937.87(-$ $\mathrm{C}-\mathrm{H}), 1626.23(\underline{\mathrm{N}=\mathrm{C}}), 1269.15(>\underline{\mathrm{C}-\mathrm{S}}-) .695 .84(\underline{\mathrm{C}-}$ Br). ${ }^{13}$ C-NMR: 55.484 (1 $\underline{\mathrm{C}}$ methoxy), 163.379, 135.804, 131.356, 130.785, 130.494, 130.232, (6들 phenyl), $\quad 130.185, \quad 128.805, \quad 188.706 \quad$ (3든 thiadiazole), 145.921 (1드 adjacent to naphthalene) ,158.898, 127.483, 127.333, 126.143, 125.127, 124.466, 120.972, 119.450, 113.851, 106.089 (10 C napthyl ring). ${ }^{1} \mathrm{H}$ NMR:3.871(3H, s, methoxy groups of naphthalene moiety),6.987(2H,s, bromo benzene),7.174(1H,s, hydrogen of thiazole moiety) ,7.762(6H,m,napthoxy moiety), $8.069(2 \mathrm{H}, \mathrm{d}, \mathrm{J}=8.8$ bromo benzene moiety), 8.158 $(1 \mathrm{H}, \mathrm{s}, \mathrm{NH}$ group adjacent to thiazole moiety),12.124(1H,s, CH adjacent to napthoxy moiety).LC-mass:, $\left[\mathrm{M}^{+}+1\right],(\mathrm{m} / \mathrm{Z})$ : 438.12/435.13.

\section{$5 \mathbf{e}$}

IR $\left(\mathrm{KBr}, \mathrm{Cm}^{-1}\right): 3308.5(>\underline{\mathrm{NH}}) 3013.92,2948.32(-\mathrm{C}-$ $\mathrm{H} \quad), 1625.50(\underline{\mathrm{N}=\mathrm{C}}), 1272.32 \quad(>\underline{\mathrm{C}-\mathrm{S}-}) .1505 .88$ $\left(\underline{\mathrm{NO}}_{2}, 4\right.$-nitro phenyl moiety) ${ }^{13} \mathrm{C}-\mathrm{NMR}: 60.522$ (1ㅡㅡ methoxy) , 163.302,151.446 147.445 140.155 135.017 134.955,(6C phenyl), 133.478132 .858 ,

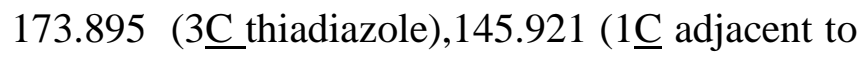
naphthalene), $153.805,132.664,131.556$, $129.315,127.887,124.300,113.702,112.642$, 111.345, $106.122 \quad\left(10 \_\mathrm{C}\right.$ napthyl ring). ${ }^{1} \mathrm{H}$ NMR:3.898 $(3 \mathrm{H}, \quad \mathrm{s}$, methoxy groups of naphthalene moiety),7.354(1H,s, hydrogen of thiazole moiety) ,7.872(6H,m,napthoxy moiety) $8.128(2 \mathrm{H}, \mathrm{d}, \mathrm{J}=8.8$ nitro benzene moiety),8.188 $(1 \mathrm{H}, \mathrm{s}, \mathrm{NH}$ group adjacent to thiazole moiety), $8.314(2 \mathrm{H}, \mathrm{d}, \mathrm{J}=8.0 \quad$ nitro benzene moiety),12.273(1H,s, $\mathrm{CH}$ adjacent to napthoxy moiety).LC-mass:,[$\left[\mathrm{M}^{+}+1\right],(\mathrm{m} / \mathrm{Z}): .405 .01$.

\section{5f}

IR $\left(\mathrm{KBr}, \mathrm{Cm}^{-1}\right): 3298.23(>\underline{\mathrm{NH}}), 3100.41,2934.05(-$ C-H stretch), 1684.07( $\underline{\mathrm{N}=\mathrm{C}}), 1260.30(>\underline{\mathrm{C}-\mathrm{S}-})$. ${ }^{13} \mathrm{C}-\mathrm{NMR}: \quad 55.267$ (1ㅁ methoxy) , 158.053, $147.332 \quad, \quad 143.445, \quad 142.190,140.675$, 134.904,(6ㄷ 3 phenyl), 129.760, 129.714,168.643 (3 $\underline{\mathrm{C}}$ thiadiazole), 146.193 (1 $\underline{\mathrm{C}}$ adjacent to naphthalene), 148.556, 128.231, 127.603, 127.407, $126.300,124.051,122.641,119.040$, 108.439, 106.395.(10 $\underline{\mathrm{C}}$ napthyl $\operatorname{ring}) .{ }^{1} \mathrm{H}$ NMR:3.898 $(3 \mathrm{H}, \quad \mathrm{s}, \quad$ methoxy groups of naphthalene moiety),7.287(1H,s, hydrogen of thiazole moiety) , $8.567(1 \mathrm{H}, \mathrm{s}, \mathrm{NH}$ group adjacent to thiazole moiety), $12.107(1 \mathrm{H}, \mathrm{s}, \mathrm{CH}$ adjacent to napthoxy moiety).8.043(4H,m, napthyl moiety) ,8.004, 8.345(2H,s, napthyl moiety ), $7.980(2 \mathrm{H}, \mathrm{s}$, methoxy napthoxy moiety) ,8.426 (2H, d, J=8.8 methoxy napthoxy moiety), 8.288, 8.304(2H, s,methoxy napthoxy moiety).LC-mass:, $\left[\mathrm{M}^{+}+1\right]$, (m/Z):409.02.

\section{$5 g$}

IR $\left(\mathrm{KBr}, \mathrm{Cm}^{-1}\right): 3308.92(>\underline{\mathrm{NH}}), 3014.21,2947.52(-$ $\mathrm{C}-\mathrm{H}), 1625.44(\underline{\mathrm{N}=\mathrm{C}}), 1270.70(>\underline{\mathrm{C}-\mathrm{S}}-), 842(\underline{\mathrm{C}}-$ Cl). ${ }^{13}$ C-NMR: 55.968 (1드 methoxy),142.234, 141.184,141.297, 137.342, 136.476, 135.562, $134.616,133.790, \quad 132.846,130.112, \quad 129.888$,

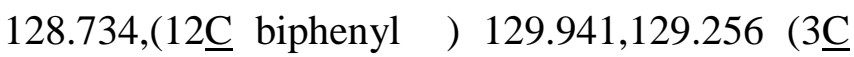
atoms of thiadiazole moiety)168.091(1 $\underline{\mathrm{C}}$ atom of $\mathrm{C}=\mathrm{N}$ in thiadiazole moiety), 145.921 (1 $\underline{\mathrm{C}}$ adjacent to naphthalene), $156.446,127.384,126.446$, $125.334,124.112,121.653,120.003,118.030$, 115.381, $111.605 \quad(10 \quad \underline{\mathrm{C}}$ napthyl $\operatorname{ring}) .{ }^{1} \mathrm{H}$ NMR:3.767 $(3 \mathrm{H}, \quad \mathrm{s}, \quad$ methoxy groups of naphthalene moiety),7.327(1H,s, hydrogen of thiazole moiety) , $8.157(1 \mathrm{H}, \mathrm{s}, \mathrm{NH}$ group adjacent to thiazole moiety), $12.071(1 \mathrm{H}, \mathrm{s}, \mathrm{CH}$ adjacent to napthoxy moiety),7.973(4H,m, napthoxy moiety), 
7.880, 7.980(2H, s, napthoxy moiety), 8.426(2H, d, J=8.8 -2,4-di chloro benzene moiety), 8.288(1H, s,- 2,4-di chloro benzene moiety).LCmass:, $\left[\mathrm{M}^{+}+1\right],(\mathrm{m} / \mathrm{Z}): 427.94 / 425.76$.

\section{5h}

IR $\left(\mathrm{KBr}, \mathrm{Cm}^{-1}\right): 3299.32(>\underline{\mathrm{NH}}), 3012.92,2954.17(-$ $\mathrm{C}-\mathrm{H}), 1625.41(\underline{\mathrm{N}=\mathrm{C}}), 1270.15$ (> $\underline{\mathrm{C}-\mathrm{S}-})$.

${ }^{13} \mathrm{C}-\mathrm{NMR}: \quad 55.784$ (1ㅁ methoxy), 164.379, 136.804, 132.356, 131.785, 131.494, 131.232, (6들 phenyl) ,131.185, $129.805,188.706 \quad$ (3든 thiadiazole), 145.981 (1드 adjacent to naphthalene) $158.898,127.483,127.333,126.143,125.127$, 124.466, 120.972, 119.450, 113.851, 106.089 . (10 $\underline{\mathrm{C}}$ napthyl ring). ${ }^{1} \mathrm{H}$ NMR:3.788 (3H, s, methoxy groups of naphthalene moiety),7.287 $(1 \mathrm{H}, \mathrm{s}$, hydrogen of thiazole moiety) , 8.347 $(1 \mathrm{H}, \mathrm{s}, \mathrm{NH}$ group adjacent to thiazole moiety), $12.098(1 \mathrm{H}, \mathrm{s}, \mathrm{CH}$ adjacent to napthoxy moiety) $.8 .043(5 \mathrm{H}, \mathrm{m}$, biphenyl moiety), 8.004(1H,s, biphenyl moiety), $8.667(3 \mathrm{H}, \mathrm{m}$, biphenyl moiety) ,7.880, 7.980(2H, s, napthoxy moiety), 8.426( $2 \mathrm{H}$, d, J=8.8 - napthoxy moiety), 8.288,8.304(2H, s, napthoxy moiety).LC-mass:, $\left[\mathrm{M}^{+}+1\right], \quad(\mathrm{m} / \mathrm{Z})$ : 436.04 .

\section{$5 \mathbf{i}$}

IR $\left(\mathrm{KBr}, \mathrm{Cm}^{-1}\right): 3304.01(>\underline{\mathrm{NH}}) 3015.16,2939.64(-$ $\mathrm{C}-\mathrm{H} \quad), 1625.42(\underline{\mathrm{N}=\mathrm{C}}), 1269.11(>\underline{\mathrm{C}-\mathrm{S}-}), 1087 \quad(\underline{\mathrm{C}-}$ F) ${ }^{13} \mathrm{C}-\mathrm{NMR}: \quad 56.168$ (1 methoxy), 141.984, $141.897,137.542,136.776,135.762,134.816$, $133.890,132.046,129.888,129.734$ (10ㅡㅡ napthyl group) ,129.141,128.256,168.091 (3 $\underline{\mathrm{C}}$ atoms of thiadiazole moiety ), 145.921 (1 $\underline{\mathrm{C}}$ adjacent to naphthalene), 151.446, $127.384,125.446,124.334$ ,123.112 , 122.653, 120.003, 119.030, 106.381, 102.605 (10 $\underline{\mathrm{C}}$ napthyl ring). ${ }^{1} \mathrm{H}$ NMR:3.898(3H, s, methoxy groups of naphthalene moiety),7.714 $(1 \mathrm{H}, \mathrm{s}$, hydrogen of thiazole moiety), 8.188 $(1 \mathrm{H}, \mathrm{s}, \mathrm{NH}$ group adjacent to thiazole moiety) ,12.274(1H,s, CH adjacent to napthoxy moiety). 7.873 (3H,m, napthoxy moiety)7.216, 7.350, $7.980(3 \mathrm{H}, \mathrm{s}$, napthoxy moiety) $8.126(2 \mathrm{H}, \mathrm{d}, \mathrm{J}=8.8$ fluoro benzene moiety), $8.288(2 \mathrm{H}, \mathrm{d}, \mathrm{J}=8.8$ fluoro benzene moiety).LC-mass: $\left[\mathrm{M}^{+}+1\right]$,

$(\mathrm{m} / \mathrm{Z})$ 378.02/377.98.

$5 \mathbf{j}$

IR $\quad\left(\mathrm{KBr}, \quad \mathrm{Cm}^{-1}\right): 3301.12(>\underline{\mathrm{NH}}), \quad 3013.14$, 2948.35(-C-H ), 1626.70( $(\underline{N}=\mathrm{C}), 1629.92(>\underline{\mathrm{C}-\mathrm{S}-}$ ). ${ }^{13} \mathrm{C}-\mathrm{NMR}: \quad 20.772,60.522$ (2들 methyl and methoxy group), $133.478132 .858,173.895$ (3든 atoms of thiadiazole moiety, 145.921 (1 $\underline{\mathrm{C}}$ adjacent to naphthalene moiety) $153.805,132.664$, $131.556,129.315,127.887,124.300,113.702$, $112.642,111.345,106.122$ (10 $\underline{\mathrm{C}}$ napthyl ring). ${ }^{1} \mathrm{H}$ NMR:2.436 $(3 \mathrm{H}, \mathrm{s}$, methyl group attached to thiazole ring), $3.879(3 \mathrm{H}, \mathrm{s}$, methoxy groups of naphthalene moiety),7.365(1H,s, hydrogen of thiazole moiety), $8.265(1 \mathrm{H}, \mathrm{s}, \mathrm{NH}$ group adjacent to thiazole moiety), $12.563(1 \mathrm{H}, \mathrm{s}, \mathrm{CH}$ adjacent to napthoxy moiety),8.013(2H,d,J=8.4, napthoxy moiety) ,7.880, 7.980(2H, s, napthoxy moiety), $8.426(2 \mathrm{H}, \mathrm{d}, \mathrm{J}=8.8$ - napthoxy moiety).LC-mass: $\left[\mathrm{M}^{+}+1\right],(\mathrm{m} / \mathrm{Z}): 297.07$.

\section{Anti- bacterial activity}

The micro-organisms were collected from the institute of microbial technology, Chandigarh, India. The antimicrobial activity of novel compounds $5 \mathrm{a}-5 \mathrm{j}$ were screened in vitro by disc diffusion method (zone of inhibition test) using Ciprofloxacin an antibiotic as a reference standard against two gram positive (Staphylococcus aureus (MTCC-7443), Bacillus subtilius (MTCC-441)) and two gram negative (Escherichia coli (MTCC725), Klebsiella pneumonia (MTCC-1739)).

The colonies of the microbial strains were inoculated on nutrient agar plates with the help of sterile loop and visually adjusted the turbidity with broth to broth to match that of $0.5 \mathrm{McF}$ arland standards. The excess of the inoculums was removed by rotating the sterile swab dipped in to the inoculum against the wall of the tube against it approximately $60^{\circ} \mathrm{C}$ between streaking, the procedure is repeated three times to ensure even distribution. After 3 mins sterile discs of the size $6 \mathrm{~mm}$ diameter were aseptically impregnated with the test compounds at a concentration $50 \mu \mathrm{g} / \mathrm{ml}$. 
The plates were incubated at $37^{\circ} \mathrm{C}$ for $24 \mathrm{~h}$. The compounds that produce distinct circular zones of inhibition around the discs the diameter of clear zone indicate the anti-bacterial activity.

\section{Antitubercular activity}

All the synthesized compounds were evaluated for their anti-tubercular activity by Micro plate Alamar Blue Assay (MABA) the bacterial strain M.tuberculosis (H37 RV strain) was used for the screening.

The 96 wells plate of outer perimeter was inoculated with $200 \mu \mathrm{l}$ of sterile water and $100 \mu \mathrm{l}$ of middle brook $7 \mathrm{H} 9$ broth and serial dilution of compound were made directly on plate The final drug concentration tested were 100 to $3.12 \mu \mathrm{g} / \mathrm{ml}$, the plates were sealed with parafilm and incubated at $37^{\circ} \mathrm{C}$ for 5 days. Later $25 \mu$ of freshly prepared mixture of Alamar Blue reagent and10\%tween 80 in 1:1 ratio was added and incubated for 24 hours .A blue colour in the well was interpreted as no bacterial growth and pink colour as bacterial growth. The antibiotic drugs such as pyrazinamide, streptomycin and ciprofloxacin was used as reference standard, whose standard values are $3.12 \mu \mathrm{g} / \mathrm{ml}, 6.25 \mu \mathrm{g} / \mathrm{ml}$ and $3.125 \mu \mathrm{g} / \mathrm{ml}$ respectively.

\section{RESULTS AND DISCUSSION}

The reagents used in the reaction were derived from the commercial sources. Melting points of the compounds (5a-5j) were determined by the open capillary method and it was uncorrected. The purity of novel compounds was confirmed by observing single spot on TLC plate, Merck silica gel $60 \mathrm{~F}_{254}$ coated alumina plates. The structures of these novel compounds $(5 \mathrm{a}-5 \mathrm{j})$ were confirmed through spectral studies. The IR spectra $\left(\mathrm{cm}^{-1}\right)$ were recorded on a Shimadzu-FTIR 577 infrared spectrometer in $\mathrm{KBr}$ pellets. The ${ }^{1} \mathrm{H}-\mathrm{NMR}$ and ${ }^{13} \mathrm{C}-\mathrm{NMR}$ spectra was recorded on Brucker AMX$400(400 \mathrm{MHz})$ spectrometer using $\mathrm{CdCl}_{3}-\mathrm{d}$ as solvent and TMS as the internal standard. The mass spectra were recorded on Perkin -Elmer
018444Y, triple quadrapole LC/MS spectrometer. The data is included in characterization Table-1. The correct sequence of the reaction scheme for the synthesis of the target compounds are shown in the figure 1.The literature studies of the thiazole derivatives indicated that the thiazole derivatives show enormous biological activities, hence inspired by this, it was decided to synthesize a series of novel thiazole derivates and screened for it various biological activities.

All the synthesized compounds were screened for the anti- bacterial activity by the disc diffusion method (ZOI test). The target compounds showed different results for the anti-bacterial screening i.e. most of the compounds exhibit satisfactory results but few of the compound showed promisingly good results.. Analysis showed that the compound $5 \mathrm{~h}$ found to be extremely sensitive towards both gram positive and gram negative bacteria for the test strains used where as compound $5 \mathrm{c}$ and $5 \mathrm{~g}$ were sensitive towards only one kind of bacterial strain and the compound $5 \mathrm{e}$ was sensitive towards only gram negative bacteria and showed maximum inhibition in case of E.coli bacterial strain. Hence the above mentioned compounds can be considerd as drug candidates for the bacterial infections. The results obtained in the anti- bacterial activity is summarized in Table2.

All the synthesized compounds were screened for the in vitro anti-tubercular activities and are presented in the Table-3.Among the tested compounds 5d showed good and remaining compounds exhibited moderate Anti- TB activity. The relative potency indicates that novel compounds (5a-5j) tested in the present study are not as effective as that of the standard compounds pyrazinamide, streptomycin and ciprofloxacin drugs but target compounds may be considered as Anti-TB agent. 
TABLE -1: CHARACTERIZATION DATA OF THE NOVEL SYNTHESISED NOVEL COMPOUNDS.

\begin{tabular}{lllcl}
\hline \hline Compd. & $\mathrm{R}$ & Mol. Formula & $\mathrm{M} . \mathrm{W}$ & $\mathrm{M} . \mathrm{P}^{0} \mathrm{C}$ \\
\hline $5 \mathrm{a}$ & $-\left(4-\left(\mathrm{OCH}_{3}\right)-\mathrm{Ph}\right)$ & $\mathrm{C}_{22} \mathrm{H}_{19} \mathrm{~N}_{3} \mathrm{O}_{2} \mathrm{~S}$ & 389.470 & $207-209$ \\
$5 \mathrm{~b}$ & $-\left(4-\left(\mathrm{CH}_{3}\right)-\mathrm{Ph}\right)$ & $\mathrm{C}_{22} \mathrm{H}_{19} \mathrm{~N}_{3} \mathrm{OS}$ & 373.470 & $217-220$ \\
$5 \mathrm{c}$ & $-(4-(\mathrm{Cl})-\mathrm{Ph})$ & $\mathrm{C}_{22} \mathrm{H}_{16} \mathrm{ClN}_{3} \mathrm{OS}$ & 393.889 & $115-118$ \\
$5 \mathrm{~d}$ & $-(4-(\mathrm{Br})-\mathrm{Ph})$ & $\mathrm{C}_{21} \mathrm{H}_{16} \mathrm{BrN}_{3} \mathrm{OS}$ & 438.340 & $222-223$ \\
$5 \mathrm{e}$ & $-\left(4-\left(\mathrm{NO}_{2}\right)-\mathrm{Ph}\right)$ & $\mathrm{C}_{21} \mathrm{H}_{16} \mathrm{~N}_{4} \mathrm{O}_{3} \mathrm{~S}$ & 404.442 & $218-221$ \\
$5 \mathrm{f}$ & $-\left(\mathrm{C}_{10} \mathrm{H}_{8}\right)$ & $\mathrm{C}_{25} \mathrm{H}_{19} \mathrm{~N}_{3} \mathrm{OS}$ & 409.502 & $178-181$ \\
$5 \mathrm{~g}$ & $-\left(3,4-(\mathrm{Cl})_{2}-\mathrm{Ph}\right)$ & $\mathrm{C}_{21} \mathrm{H}_{15} \mathrm{Cl}_{2} \mathrm{~N}_{3} \mathrm{OS}$ & 428.334 & $204-206$ \\
$5 \mathrm{~h}$ & $-(\mathrm{Ph}-\mathrm{Ph})$ & $\mathrm{C}_{27} \mathrm{H}_{21} \mathrm{~N}_{3} \mathrm{OS}$ & 435.540 & $136-137$ \\
$5 \mathrm{i}$ & $-(4-(\mathrm{F})-\mathrm{Ph})$ & $\mathrm{C}_{21} \mathrm{H}_{16} \mathrm{FN}_{3} \mathrm{OS}$ & 377.434 & $242-250$ \\
$5 \mathrm{j}$ & $-\mathrm{CH}$ & $\mathrm{C}_{16} \mathrm{H}_{15} \mathrm{~N}_{3} \mathrm{OS}$ & 297.374 & $238-240$ \\
\hline
\end{tabular}

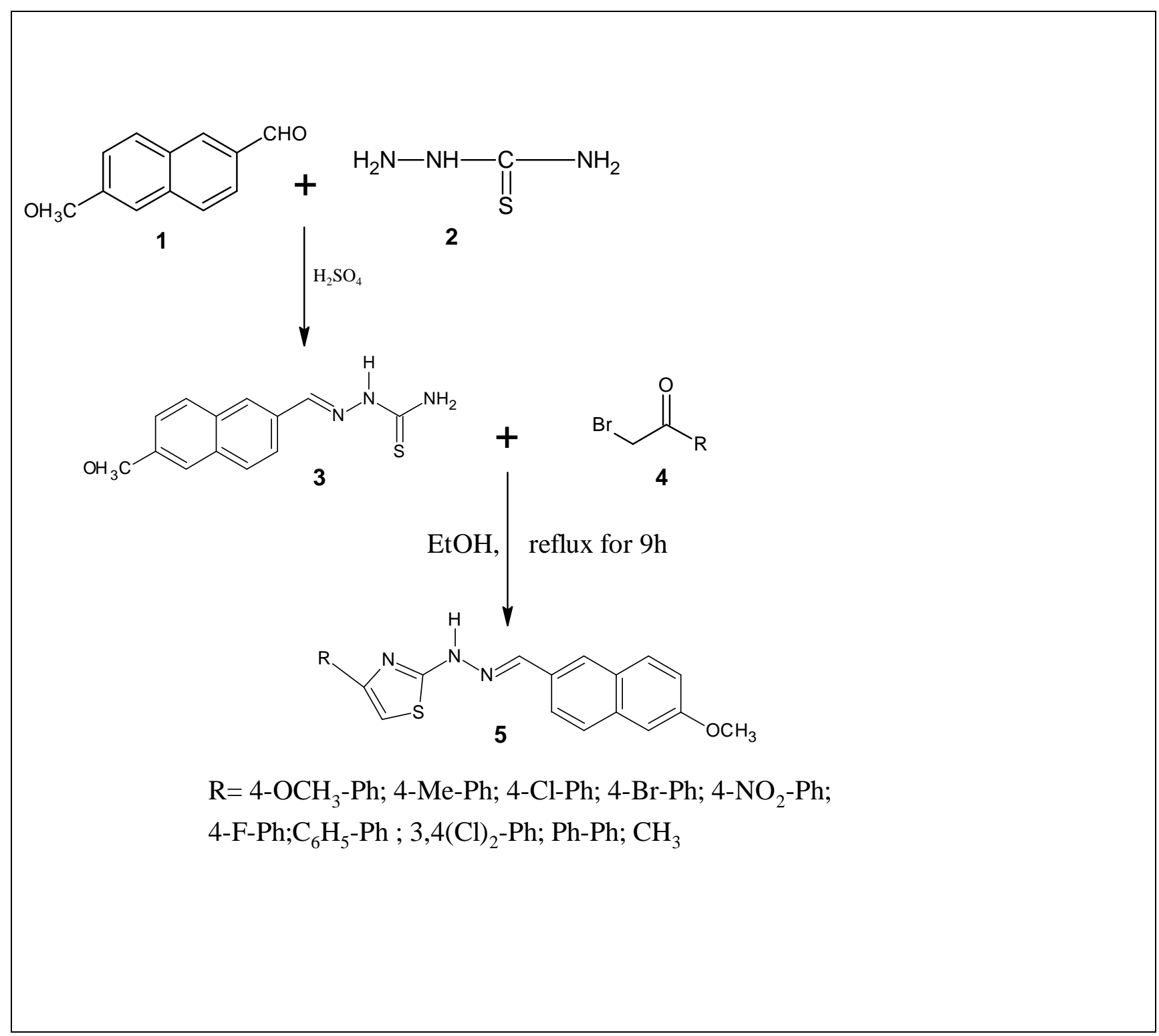

Figure 1: Synthetic route for the preparation of target compounds (5a-5j) 
TABLE -2: ANTI-BACTERIAL ACTIVITY OF STANDARD AND TEST COMPOUNDS.

\begin{tabular}{lllll}
\hline \hline Compd. & \multicolumn{4}{c}{ Diameter of inhibition Zone } \\
\hline & Gram positive bacteria & \multicolumn{2}{l}{ Gram negative bacteria } \\
\hline & $\begin{array}{l}\text { Staphylococcus } \\
\text { aureus }\end{array}$ & Bacillus subtilius & Escheric & Klebsiella \\
& $11 \mathrm{~mm}$ & $12 \mathrm{~mm}$ & $16 \mathrm{~mm}$ & $12 \mathrm{~mm}$ \\
\hline $5 \mathrm{a}$ & $10 \mathrm{~mm}$ & $20 \mathrm{~mm}$ & $12 \mathrm{~mm}$ & $19 \mathrm{~mm}$ \\
$5 \mathrm{~b}$ & $20 \mathrm{~mm}$ & $15 \mathrm{~mm}$ & $30 \mathrm{~mm}$ & $22 \mathrm{~mm}$ \\
$5 \mathrm{c}$ & $16 \mathrm{~mm}$ & $11 \mathrm{~mm}$ & $10 \mathrm{~mm}$ & $12 \mathrm{~mm}$ \\
$5 \mathrm{~d}$ & $16 \mathrm{~mm}$ & $13 \mathrm{~mm}$ & $27 \mathrm{~mm}$ & $18 \mathrm{~mm}$ \\
$5 \mathrm{e}$ & $11 \mathrm{~mm}$ & $9 \mathrm{~mm}$ & - & $19 \mathrm{~mm}$ \\
$5 \mathrm{f}$ & $22 \mathrm{~mm}$ & $28 \mathrm{~mm}$ & $29 \mathrm{~mm}$ & $17 \mathrm{~mm}$ \\
$5 \mathrm{~g}$ & $21 \mathrm{~mm}$ & $22 \mathrm{~mm}$ & $26 \mathrm{~mm}$ & $24 \mathrm{~mm}$ \\
$5 \mathrm{~h}$ & $10 \mathrm{~mm}$ & $14 \mathrm{~mm}$ & $12 \mathrm{~mm}$ & $16 \mathrm{~mm}$ \\
$5 \mathrm{i}$ & $12 \mathrm{~mm}$ & $16 \mathrm{~mm}$ & $16 \mathrm{~mm}$ & $16 \mathrm{~mm}$ \\
$5 \mathrm{j}$ & $26 \mathrm{~mm}$ & $30 \mathrm{~mm}$ & $32 \mathrm{~mm}$ & $28 \mathrm{~mm}$ \\
Ciprofloxacin & & & & \\
\hline
\end{tabular}

${ }^{A}$ Mean values of 3 trails. ' 0 ' indicates no sensitivity (zone of inhibition $<7 \mathrm{~mm}$ )

TABLE-3: ANTI-TUBERCULAR ACTIVITY OF THE SYNTHESIZED COMPOUNDS AGAINST REFERNCE STANDARDS.

\begin{tabular}{lllllll}
\hline \hline SAMP & 100 & 50 & 25 & 12.5 & 6.25 & 3.12 \\
LES & $\mu \mathrm{g} / \mathrm{ml}$ & $\mu \mathrm{g} / \mathrm{ml}$ & $\mu \mathrm{g} / \mathrm{ml}$ & $\mu \mathrm{g} / \mathrm{ml}$ & $\mu \mathrm{g} / \mathrm{ml}$ & $\mu \mathrm{g} / \mathrm{ml}$ \\
\hline & & & & & & \\
$5 \mathrm{a}$ & $\mathrm{S}$ & $\mathrm{S}$ & $\mathrm{R}$ & $\mathrm{R}$ & $\mathrm{R}$ & $\mathrm{R}$ \\
$5 \mathrm{~b}$ & $\mathrm{~S}$ & $\mathrm{~S}$ & $\mathrm{R}$ & $\mathrm{R}$ & $\mathrm{R}$ & $\mathrm{R}$ \\
$5 \mathrm{c}$ & $\mathrm{S}$ & $\mathrm{S}$ & $\mathrm{S}$ & $\mathrm{R}$ & $\mathrm{R}$ & $\mathrm{R}$ \\
$5 \mathrm{~d}$ & $\mathrm{~S}$ & $\mathrm{~S}$ & $\mathrm{~S}$ & $\mathrm{~S}$ & $\mathrm{~S}$ & $\mathrm{R}$ \\
$5 \mathrm{e}$ & $\mathrm{S}$ & $\mathrm{S}$ & $\mathrm{R}$ & $\mathrm{R}$ & $\mathrm{R}$ & $\mathrm{R}$ \\
$5 \mathrm{f}$ & $\mathrm{S}$ & $\mathrm{S}$ & $\mathrm{R}$ & $\mathrm{R}$ & $\mathrm{R}$ & $\mathrm{R}$ \\
$5 \mathrm{~g}$ & $\mathrm{~S}$ & $\mathrm{~S}$ & $\mathrm{R}$ & $\mathrm{R}$ & $\mathrm{R}$ & $\mathrm{R}$ \\
$5 \mathrm{~h}$ & $\mathrm{~S}$ & $\mathrm{~S}$ & $\mathrm{R}$ & $\mathrm{R}$ & $\mathrm{R}$ & $\mathrm{R}$ \\
$5 \mathrm{i}$ & $\mathrm{S}$ & $\mathrm{S}$ & $\mathrm{R}$ & $\mathrm{R}$ & $\mathrm{R}$ & $\mathrm{R}$ \\
$5 \mathrm{j}$ & $\mathrm{S}$ & $\mathrm{S}$ & $\mathrm{R}$ & $\mathrm{R}$ & $\mathrm{R}$ & $\mathrm{R}$ \\
& & & & & & \\
\hline
\end{tabular}

S-Sensitive R-Resistant 


\section{CONCLUSION}

The screening studies of the antibacterial and antitubercular activity studies of the synthesized novel compounds proved to be potent agents for the respective studies. In conclusion, a series of novel thiazoles were synthesized which were analyzed for anti-bacterial and anti-tubercular activities. Presence of electron withdrawing groups like Chlorine and nitro groups at the para position of the phenyl ring attached to the thiazole nucleus as substituent are responsible for the good antibacterial and moderate anti-tubercular activity. A further study of these compounds with special reference to therapeutic index for the drug is going on.

\section{ACKNOWLEDGEMENTS}

The authors express their heartfelt thanks to, The head ,NMR research center ,SAIF - cochin-22 for 1H-NMR,13C-NMR spectroscopy and FT-IR analysis, Syngenta Goa for Mass spectroscopy, Maratha mandal's NGH institute of dental sciences and research center, Belgaum for Microbiological activities.

\section{REFERENCE}

1. Rahul.D.K, Rohan.J.M, Shrikant.V. H, Rahul.A.M, Sonali.S.K, Rajesh.N.G, et al. Synthesis and in silico investigation of thiazoles bearing pyrazoles derivatives as anti-inflammatory agents. Comp. Biol. Chem 2016; 61:86-96.

2. Satish.K, Deepika.R.K.C, Sreenivas.A, Jayaram.R.K, Srigiridhar.K, Rambabu.Y. Synthesis and anticancer evaluation of 3aryl-6-phenylimidazo [2,1-b]thiazoles. Bioorg. Med. Chem. Lett 2014; 24:54285431.

3. Carlos Nava.Z, Fabiola.C, Rosa.M, Manuel.J.C, Benjamin.O.M, Hermenegilda. M.D, et al.. 2-Acylamino-5-nitro1,3-thiazoles: Preparation and in vitro bioevaluation against four neglected protozoan parasites. Bioorg. Med. Chem 2014; 22:1626-1633.
4. Aldo.A, Alberto.L, Alessandra.L, Rita.M, Mirella.R, Rinaldo.C et al. Thiazoles. Eur. J. Med. Chem 2013; 68:412-421.

5. Nataliya.Z, Dmytro.A, Olexandr.V, Philippe.G, Roman.L. Thiazoles. Bioorg. Med. Chem. Lett 2012; 22:7071-7074.

6. Belem.A, Aaron.R, Heather.S, Donard .S.D, Mark.J.K. Triazolbenzo[d]thiazoles: Efficient synthesis and biological evaluation as neuroprotective agents , Bioorg. Med. Chem. Lett 2012; 22:5976-5978.

7. Maria.I.L.S, Ana.F.B, Mafalda.L, Jose. A.P, Filomena.M.B, Teresa.M.V.D, et al. Chiral 6,7-bis(hydroxymethyl)-1H,3Hpyrrolo[1,2-c]thiazoles with anti-breast cancer properties . Eur. J. Med. Chem 2013; 60: 254-262.

8. Nareshvarma.S, Shrivastava.S.P. Synthesis and in vitro study of $[1,3,4]$ thiadiazol-2yl3,3a,5,6-tetrahydro-2H-pyrazolo[3,4d]thiazoles as antimicrobial agents Journal of Saudi Chemical Society 2016;20:33-39.

9. Shahenda.M. E, Ghada.S.H, Fatmah.A.M. A, Hussein.I.E. Substituted thiazoles VI. Synthesis and antitumor activity of new 2acetamido- and 2 or 3-propanamidothiazoleanalogs. Eur. J. Med. Chem.Volume 2012; 54:615-625.

10. Ghada.S.H, Shahenda.M.E, Fatmah. A.M.A, Hussein.I.E. Substituted thiazoles VII. Synthesis and antitumor activity of certain 2-(substituted amino)-4-phenyl-1,3-thiazole analogs, Bioorg. Med. Chem. Lett 2012; 22:63186323.

11. Kalkhambkar.R.G, Kulkarni.G.M, Shivkumar. H, Nagendra.R.R. Synthesis of novel triheterocyclic thiazoles as antiinflammatory and analgesic agents, Eur. J. Med. Chem 2007; 42:1272-1276.

12. Nadee.S, Waquar.A. Triazole incorporated thiazoles as a new class of anticonvulsants: Design, synthesis and in vivo screening. Eur. J. Med. Chem 2010; 45:1536-1543. 
13. Nathan.A, Axerio.C.P, Tavassoli, Peyman, Han, Frank.Q, et al. Targeting the Binding Function 3 (BF3) Site of the Human Androgen Receptor through Virtual Screening. J. Med. Chem 2011; 54:8563-8573.

14. Garcia-Domenech, Ramon, Barbosa, Luciana, Lacarra, Matilde, et al. Application of molecular topology to predict the inhibition of Trypanosoma cruzi cruzain by thiosemicarbazones. J. Mol.Design 2008; 7:260-272.

15. Cohen, Fred.E, Du, Xiaohui.G , Chun, Mckerrow, James H. Preparation of thiosemicarbazones and semicarbazones as inhibitors of cysteine proteases and methods of their use. U.S. Pat. Appl. Publ. 2004, US 20040014801 A12004012

2.

16. Du, Xiaohu, Guo, Chun, Hansell, Elizabeth, et al. Synthesis and StructureActivity Relationship Study of Potent Trypanocidal Thio Semicarbazone Inhibitors of the Trypanosomal Cysteine Protease Cruzain. J. Med. Chem 2002; 45:2695-2707.

17. Zee-Cheng, Kwang.Y, Nyberg, Wayne.H, Cheng.C.C. Synthesis of 8,9-dialkoxysubstituted tetrahydrobenz[h]isoquinolines. J. Heterocyclic Chem 1972; 9: 805-11.

18. Du, Xiaohui. Synthesis and StructureActivity Relationship Study of Potent Trypanocidal Thio Semicarbazone Inhibitors of the Trypanosomal Cysteine Protease Cruzain. J. Med. Chem 2002; 45:2695-2707.

19. Cohen, Fred. E. Preparation of thiosemicarbazones and semicarbazones as inhibitors of cysteine proteases and methods of their use. U.S. Pat. Appl. Publ.20040014801, 2004. 